\title{
Large-Scale Text to Image Retrieval Using a Bayesian $K$-Neighborhood Model ${ }^{\star}$
}

\author{
Roberto Paredes \\ ITI-UPV \\ Camino de Vera S/N, 46022 Valencia (Spain)
}

\begin{abstract}
In this paper we introduce a new approach aimed at solving the problem of image retrieval from text queries. We propose to estimate the word relevance of an image using a neighborhood-based estimator. This estimation is obtained by counting the number of word-relevant images among the $K$-neighborhood of the image. To this end a Bayesian approach is adopted to define such a neighborhood. The local estimations of all the words that form a query are naively combined in order to score the images according to that query. The experiments show that the results are better and faster than the state-of-theart techniques. A special consideration is done for the computational behaviour and scalability of the proposed approach.
\end{abstract}

\section{Introduction}

This paper addresses the problem of image retrieval from text queries. This problem is commonly treated and it is a fundamental part of web search engines and photographic databases. Image retrieval from text is a particular example of an information retrieval system where the user uses text queries in order to search for the requested information. Therefore the methodology proposed here could be easily applied to other scenarios such as video and audio retrieval. The precision of the image retrieval systems has been improved during the last years due to the introduction of new image descriptors and methodologies. In the case of web search engines the current image retrieval technology is mainly based on the text that appears around the images in the web pages. On the other hand, in online photographic databases like Flicker or Picasa, the textual information related to the images is extracted from the tags with which the user described the pictures during the uploading process. Despite of the straightforward implementation and relatively good results of such an approach, it can not be extended to other scenarios where that textual information is not available. The problem of image retrieval from text queries is usually solved by means of ranking the images according to their relevance to the query meaning. The images are sorted with regard to the scores that they obtain for a particular query and the images with the highest scores are presented to the user. Therefore the retrieval problem is reduced to the computation of the scores for any pair query-image.

\footnotetext{
* Work supported by the UPV project PAID09015 and Spanish projects: TIN2008-04571 and Consolider Ingenio 2010 MIPRCV (CSD2007- 00018).
} 
Recently different approaches for solving this problem using a neighborhood model have been proposed [13 9]. In [13] the authors proposed two different ways of linearly combining different distances that define the image neighborhood. In [9] the authors propose two different approaches, weighted nearest neighbor for tag prediction and word-specific logistic discriminant models. These methods show the capabilities of the neighborhood-based estimators to solve the image annotation problem, but these methods scale poorly and can not be applied to large scale problems with a huge number of images (millions) and large size of the vocabulary (thousands).

For large scale problems a linear discriminative model (PAMIR) has been proposed in order to rank images from text queries [8]. This method outperforms other techniques like Cross-Media Relevance Model (CMRM) [11], Cross-Media Translation Table (CMTT) [17], Probabilistic Latent, Semantic Analysis (PLSA) [15] and Support Vector Machines (SVM) [16],[19]. This approach has demonstrated good performance and a good scalability behaviour following an online learning approach. This method can be considered the state-of-the-art for large scale image retrieval from text queries. The current paper proposes a new approach based on a local word relevance estimation. This local estimation is accomplished by considering the $K$-neighborhood of the images but the present work aims at guaranteeing the scalability capabilities.

The paper is organized as follows. The new approach is presented in section 2 Computational issues are considered in section 3 Experiments with two different datasets are carried out in section 4 . Finally, some conclusions are drawn in section 5.

\section{Approach}

In this section the new approach based on a $K$-neighborhood word relevance estimation is presented. The new method will be denoted as KNIR ( $K$-Neighborhood Image Retrieval).

In order to retrieve images given a text query, a score for any image given this text query is needed. This score should be high when the image content is relevant to the text query and should be low when the image content is not relevant to the text query.

Given an image $\mathbf{p}$ and a text query $q$ represented by a bag of words $q=\left\{w_{1}, w_{2}, \ldots\right.$, $\left.w_{n}\right\}$. We propose the following score

$$
s c(q, \mathbf{p})=p(q \mid \mathbf{p})
$$

A linearly smoothed naive Bayes decomposition of $p(q \mid \mathbf{p})$ yields:

$$
p(q \mid \mathbf{p})=\prod_{i=1}^{n} p\left(w_{i} \mid \mathbf{p}\right)=\prod_{i=1}^{n}\left(\beta \hat{p}\left(w_{i} \mid \mathbf{p}\right)+(1-\beta) \frac{1}{|d|}\right)
$$

where $d$ is the size of the text vocabulary.

The expression to estimate is $\hat{p}\left(w_{i} \mid \mathbf{p}\right)$, an estimation of the conditional probability of the word $w_{i}$ given the image $\mathbf{p}$. To estimate this conditional distribution we define the set $P_{i}^{+}$. This set is the set of pictures that are relevant to queries where the word $w_{i}$ appears. 
The following $K$-neighborhood estimator is proposed:

$$
\hat{p}\left(w_{i} \mid \mathbf{p}\right)=\frac{C_{K i}}{K}
$$

where $C_{K i}$ is the number of pictures that belong to $P_{i}^{+}$among the $K$-nearest of $\mathbf{p}$.

\subsection{Parameter Selection}

To compute the proposed score we have to define two parameters $K$ and $\beta$. In the experiments the parameter $\beta$ was found not to be critical. On the other hand, the parameter $K$ has an important impact since this parameter defines the image neighborhood considered. Here we are going to focus on this parameter and how to estimate it adequately. Instead of trying different values of this parameter and selecting the best one using a validation set, a Bayesian approach is proposed:

$$
p\left(w_{i} \mid \mathbf{p}\right)=\sum_{\forall K} p\left(w_{i} \mid \mathbf{p}, K\right) p(K \mid \mathbf{p})
$$

The term $p\left(w_{i} \mid \mathbf{p}, K\right)$ is what we are estimating in equation 3 for a particular value of $K$. Therefore applying this Bayesian approach to our problem and limiting the values of $K$ to some maximum value $K_{\max }$, equation 4 can be expressed as:

$$
\hat{p}\left(w_{i} \mid \mathbf{p}\right) \approx \sum_{K=1}^{K_{\max }} \frac{C_{K i}}{K} p(K \mid \mathbf{p})
$$

Here, usually, a Markov chain Monte Carlo (MCMC) procedure is used in order to draw parameters from the $p(K \mid \mathbf{p})$ distribution, selecting those parameters with the highest likelihood, see for instance [6] and [14]. In this work a more simple, yet effective approach is proposed by assuming an uniform distribution of the parameter space: $p(K \mid \mathbf{p}) \sim U\left(1, K_{\max }\right)$. Therefore equation 5 becomes:

$$
\hat{p}\left(w_{i} \mid \mathbf{p}\right) \approx \frac{1}{K_{\max }} \sum_{K=1}^{K_{\max }} \frac{C_{K i}}{K}
$$

and finally equation 2 can be rewritten as:

$$
p\left(w_{i} \mid \mathbf{p}\right) \approx \beta \frac{1}{K_{\max }} \sum_{K=1}^{K_{\max }} \frac{C_{K i}}{K}+(1-\beta) \frac{1}{|d|}
$$

The parameter $K_{\max }$ is indeed a parameter to be tuned but, as the experiments will show, this parameter leads to better results than the parameter of the conventional $K$ neighborhood in a wide range of values. It is important to note that the Bayesian approach does not entail more computations than the standard one. The Bayesian approach only requires to define the $K_{\max }$-neighborhood. Only one search is needed, and then the votes of each image among the $K_{\max }$-nearest are adequately weighted depending on the rank obtained. That is, nearest images have higher weight while farther images have lower weight. 
The Bayesian approach and the naive Bayes decomposition of the query lead to the following expression of the required score:

$$
s c(q, \mathbf{p})=\prod_{i=1}^{n} \beta \frac{1}{K_{\max }} \sum_{K=1}^{K_{\max }} \frac{C_{K i}}{K}+(1-\beta) \frac{1}{|d|}
$$

\section{Computational Issues}

In this section we discuss the computational issue to be considered in order to achieve a good computational behaviour and scalability properties.

First of all it is important to distinguish between training and test images. The difference between training and test images is that for the training images we know the relevance judgment for a set of training queries while for the test images we do not have such judgment. This is an important issue to take into account because in a practice scenario the number of images whose description is known uses to be very reduced in comparison with the total number of images available. However we aim at performing the image retrieval process over all the images instead of over the annotated set only. Moreover in order to evaluate the generalization capabilities of the approaches, training images used to be discarded and the image retrieval used to be performed over nonannotated images. Therefore the training images are used to define the sets $P_{i}^{+}$while for the test images, $\mathbf{p}$, we have to estimate the conditional probability $p\left(w_{i} \mid \mathbf{p}\right)$ for any word of the vocabulary. This conditional probability is estimated in training time and it is stored in a table for each pair $\left(w_{i}, \mathbf{p}\right)$. The computation of the score $\operatorname{sc}(q, \mathbf{p})$ is reduced to the product of the values that appear in this table in the positions corresponding to the words that belong to that query and that particular image. This process is performed in test time for a given query.

An efficient implementation of the computation of the term $p\left(w_{i} \mid \mathbf{p}\right)$ is required in order to achieve a fast training time and scalability. Two important components affect this computational behaviour. First, to obtain the set $P_{i}^{+}$for every word $w_{i}$ of the vocabulary. Second, to compute the $K$-neighborhood of any image p. Clearly the most costly part is the computation of the neighborhood where the distances between $\mathbf{p}$ and all the training images must be obtained. To this end fast search algorithms can be used in order to alleviate such task. Although different techniques have been tested, here only the best one are described. Furthermore, since only vectorial representation of the images have been considered, only vectorial approaches have been tested. Two different approaches have been finally used depending on the dataset, Visual Word Hashing (VWH) and Local Sensitive Hashing (LSH) [7]. The approach that uses fast search algorithm will be denote as FKNIR (Fast KNIR).

\section{Visual Word Hashing}

The first dataset used in the experiments is the same dataset that was used in [8]. In this dataset the image representation is very sparse and the well known LSH does not provide the best computational performance. Due to the sparsity of the image representation VWH has been proposed. VWH is a very simple method, the idea is to store 
each image in several buckets. The maximum number of buckets is $D^{2}$ being $D$ the number of visual words of the image representation, so $\mathbf{p} \in \Re^{D}$. Therefore each bucket stores the indexes of the images with a particular pair of visual words. The buckets usually form a sparse matrix and standard hashing is used to deal with such data structure. Higher order visual word correlations could be used but the second order leads to good enough results.

\section{Local Sensitive Hashing}

The image representation used on the second experiments does not lead to an important sparsity of the data. In this case the well known LSH [7] lead to the best performance. To this end $r$-stable-random projections of the image $\mathbf{p}$ are computed [10]. The projection value then is binarized depending on its sign. These $r$ binary numbers are randomly selected forming $w$ different words of $b$ bits, clearly $b<r$ must be fulfilled, $b \ll r$ is advisable. Finally the index of the image $\mathbf{p}$ is stored in the buckets associated to the $w$ words. So it is expected that this method split the image representation space into $2^{b}$ buckets but each image can appear in up to $w$ different buckets.

In both methods the search of the $K$-neighbors for a test image is performed computing the original distance (usually $L_{p}$ family) between the image and all the images that fall into the same buckets.

\section{Experiments}

The experiments have been carried out with two different datasets, Corel and ImageClef. Both data sets are split into development and test set. The development set is further split into training and evaluation. Each partition contains pictures, text queries and the relevance judgment for any pair picture-query. The evaluation set is used for tuning the model parameters and the test set is finally used to evaluate the different models. This test set evaluation is performed by means of two measures: average precision (AvgP) and precision at top 10 (P10) both in percentage. The training time of the different methods is also measured.

In general in all the experiments the validation set was used to tune the parameters of the different methods. The parameter $C$ of PAMIR was varied from 0.001 to 1.0 and the number of iterations from $10^{5}$ to $10^{8}$. The parameter $K_{\max }$ of the KNIR method was varied from 10 to 1000 .

The results obtained with our implementation of PAMIR are almost identical to the results reported in [8].

\subsection{Corel Dataset}

These experiments were carried out using the same dataset used in [8]. In fact preprocesed version of this dataset was provided by the authors of [8] so an exact comparison can be made. This data is composed by two different partitions, Corel-small and Corellarge. Both sets originate from the Corel stock photography collection, which offers a large variety of pictures, ranging from wilderness scenes to architectural building pictures or sport photographs. 


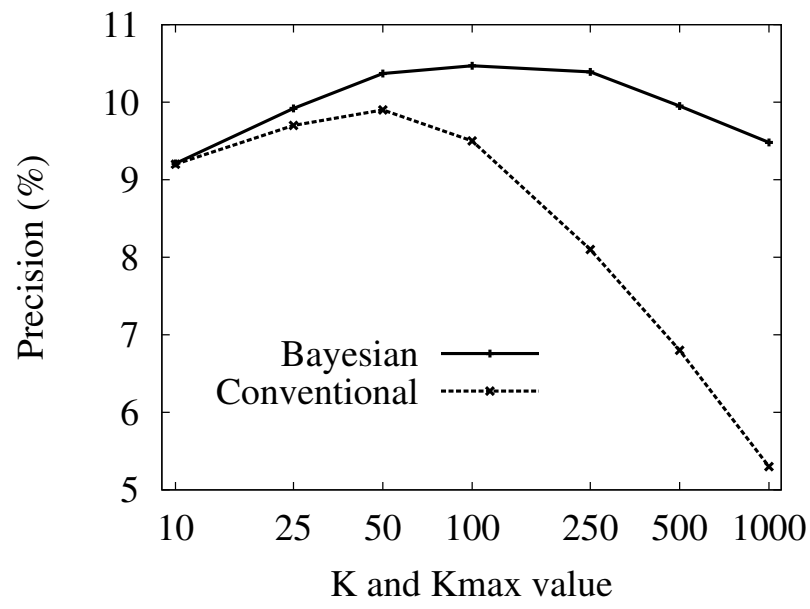

Fig. 1. Comparison between conventional K- neighborhood and Bayesian K-Neighborhood performance on the Corel-small validation dataset

One common issue in both partitions is that the images are represented using a very high dimensional vectorial representation; namely, 10,000 components that come from the concatenation of two different sets of image features: Local Binary Patterns and Color histograms. This high dimensional representation is not a casual selection but this high dimensional representation is somewhat required by the PAMIR approach. This approach relies on the linear separation of the image representation space for a given word. That is, the image representation space should be linearly split into the relevant and not-relevant images for a given word. This linear separation is not fully accomplished when the image representation selected has not such very high dimensionality. In this sense, PAMIR requires that the practitioners use such high dimensionality representation reducing in some situations the practitioners choices.

Corel-small dataset. Corel-small corresponds to the 5,000-picture set presented in [4]. This set, along with the provided split between development and test data, has been used extensively in the query-by-text literature, e.g. [1], [12],[15]. The development set is composed by 4,500 pictures that are further split into 4,000 pictures for training and 500 pictures for evaluation. The test set is composed by 500 pictures. The number of queries are 7,221,1,962 and 2,241 for training, validation and test respectively.

Figure 1 shows the precision of the retrieval system comparing the Bayesian and the conventional neighborhood for different values of $K_{\max }$ and $K$ respectively. The Bayesian approach shows a better behaviour and the selection of parameter $K_{\max }$ is less critical than the selection of parameter $K$ for the conventional approach.

Table 1 shows the results obtained for the small dataset. The precision, average precision and training time are compared for PAMIR, KNIR and FKNIR. As commented before, FKNIR uses a VWH method, similar but slower results were obtained using LSH. It is important to note that the sparsity of the image representation is a very 
Table 1. Results on Corel-small test set

\begin{tabular}{|l|c|c|c|}
\hline Method & P@ 10(\%) & AvgP (\%) & Training (ms) \\
\hline \hline PAMIR & 9.97 & 25.8 & 2937 \\
KNIR & 9.9 & 26.7 & 524 \\
FKNIR & 10.0 & 27.1 & 1571 \\
\hline
\end{tabular}

Table 2. Results on Corel-large test dataset

\begin{tabular}{|l|c|c|c|}
\hline Method & P@ 10(\%) & AvgP (\%) & Training (secs) \\
\hline \hline PAMIR & 2.69 & 4.98 & 63.7 \\
KNIR & 2.73 & 4.78 & 26.9 \\
FKNIR & 2.84 & 4.92 & 18.2 \\
\hline
\end{tabular}

important factor in the computational cost of the algorithms. Thus, depending on the sparsity of the dataset the selection of the fast search algorithm could be reconsider.

The results obtained for the KNIR methods and PAMIR are almost identical. Only the average precision results of KNIR methods show some small improvements. The computation time of KNIR is clearly better than the PAMIR. In these experiments FKNIR has an important overhead, to obtain the buckets of the training images, and the time results showed in table 1 are not better than the standard KNIR, but a slight average precision improvement is obtained.

Corel-large dataset. Corel-large was proposed in [8] and contains 35,379 images and corresponds to a more challenging retrieval problem than Corel-small. This dataset is split into development and test partitions. The development set is composed by 25,120 pictures that are further split into 14,861 training pictures and 10,259 evaluation pictures. The test set is composed of 10,259 pictures. The number of queries are 55,442 , 39,690 and 39,613 for training, validation and test respectively.

Table 2 shows the results obtained for the large dataset. The precision, average precision and training time are compared for PAMIR, KNIR and FKNIR.

The results obtained for the KNIR methods and PAMIR are again almost identical. The computation time of KNIR is clearly better than the PAMIR while the FKNIR is now faster than KNIR.

These two experiments do not show a significant improvement on the precision of the here proposed approaches. On the other hand, the faster computation has been demonstrated. It seems that the Corel dataset, more concretely the image representation used in [8] has some properties that fulfill the PAMIR restrictions, that is, to be able to define an hyperplane to split the image representation space into two different regions, relevant an no relevant for a given word. Then, the $K$-neighborhood model proposed here does not have anything to add and any further improvement can be achieved in this sense.

The following experiments are conducted to show that in some circumstances the linear separation of the image representation space is not completely fulfilled and the here proposed approach can provide improvements. 


\subsection{ImageCLEF Dataset}

This experiment was conducted using the ImageClef photo annotation dataset [5]. The total of 20,000 images were split into 18,000 training images, 1,000 validation images and 1,000 test images. A small vocabulary of 124 words was extracted from the image annotations. A total number of 10,647, 1,572 and 1,610 queries were generated for the training, validation and test sets respectively. The validation set was used to adjust the parameters as in the previous experiments.

The images were represented using two different features: Local image descriptors and color histograms. In this work the local image descriptors are patches that are extracted from the images at regular grid positions and dimensionality reduced using PCA transformation [3]. These local descriptors are finally represented using an histogram of visual words [2], [18]. On the other hand, color histograms are among the most basic approaches, widely used in image retrieval and it gives reasonably good results. The color space is partitioned and for each partition the pixels with a color within its range are counted, resulting in a histogram representation.

As mentioned before, PAMIR works better as the dimensionality of the image representation grows. To this end we have selected two different images representation. The first, small one, is composed of 512 visual words and 512 color histogram bins, leading to a total of 1024 dimensions. The second, large one, is composed of 4092 visual words and 512 color histogram bins, leading to a total of 4604 dimensions.

In this experiment LSH was used for the FKNIR approach. The LSH parameters were tuned using the validation dataset, selecting those parameters that show a good balance between speed and precision. The parameters were set to $r=100, w=100$ and $b=14$. One more experiment is carried out tuning the parameters of LSH in order to measure the time needed to obtain similar precision results as with PAMIR. This approach is referred as FKNIR*.

Table 3 and 4 show the results obtained for the small and large datasets respectively. In both experiments the KNIR methods clearly outperform PAMIR in both precision and speed. As expected, the results of PAMIR are better for higher dimensions but still the precision at top 10 is far from the KNIR method which represents a $30 \%$ of relative improvement over PAMIR. Moreover the computational time of PAMIR is clearly higher than in the here proposed methods. The difference between PAMIR and FKNIR* training time is remarkable. It is important to note that the results of KNIR are almost identical in both image representations, so small (and fast) image representations are enough in order to obtain good results using the here proposed model.

Table 3. ImageCLEF results for the 512-512 representation

\begin{tabular}{|l|c|c|c|}
\hline Method & P@ 10(\%) & AvgP (\%) & Training (secs) \\
\hline \hline PAMIR & 3.0 & 9.33 & 96 \\
KNIR & 4.1 & 11.93 & 35 \\
FKNIR & 3.8 & 11.27 & 8 \\
\hline FKNIR* & 3.3 & 9.0 & 4 \\
\hline
\end{tabular}


Table 4. ImageCLEF results for the 4096-512 representation

\begin{tabular}{|l|c|c|c|}
\hline Method & P@ 10(\%) & AvgP $(\%)$ & Training (secs) \\
\hline \hline PAMIR & 3.1 & 10.3 & 196 \\
KNIR & 4.0 & 11.62 & 45 \\
FKNIR & 3.9 & 11.78 & 16 \\
\hline FKNIR* & 3.7 & 10.5 & 9 \\
\hline
\end{tabular}

Comparing these results with the results obtained in Corel-large, the PAMIR behaviour is slower due to the sparsity of the image representation. In Corel the images have 40 non-zero visual words in average while in ImageClef-large the images have more than 300 non-zero visual words in average. Another important computational issue that affects PAMIR is the number of iterations of the online learning approach. However the computational cost of the here proposed approach is fixed and does not depend on an iterative procedure at all.

\section{Conclusions}

The here proposed approach has shown to be effective for the problem of image retrieval from text queries. The results obtained show that KNIR outperform the state-of-theart techniques while preserving a very good computational behaviour. The Bayesian approach adopted has shown to be very appropriate for this particular problem and does not entail any complex learning stage neither more computations on the test phase. The fast implementation of KNIR, FKNIR, obtains further computational benefits while keeping the precision performance on similar values than KNIR. On the other hand, KNIR can be used in applications where the image representation has to keep some structural information. In this sense the proposed approach only requires to be able to compute distances between the objects represented. Moreover KNIR do not require any particular high dimensional representation of the images to be effective.

\section{References}

1. Barnard, K., Duygulu, P., de Freitas, N., Forsyth, D., Blei, D., Jordan, M.I.: Matching words and pictures. Journal of Machine Learning Research 3, 1107-1135 (2003)

2. Csurka, G., Dance, C., Fan, L., Willamowski, J., Bray, C.: Visual categorization with bags of keypoints. In: ECCV (2004)

3. Deselaers, T., Keysers, D., Ney, H.: Discriminative training for object recognition using image patches. In: IEEE Conference on Computer Vision and Pattern Recognition (CVPR 2005), pp. 157-162 (2005)

4. Duygulu, P., Barnard, K., de Freitas, N., Forsyth, D.: Object recognition as machine translation: Learning a lexicon for a fixed image vocabulary. In: Seventh European Conference on Computer Vision, vol. IV, pp. 97-112 (2002)

5. Escalante, H.J., Hernández, C., Gonzalez, J., López, A., Montes, M., Morales, E., Sucar, E., Grubinger, M.: The segmented and annotated iapr tc-12 benchmark. Computer Vision and Image Understanding (2009) 
6. Everson, R.M., Fieldsend, J.E.: A variable metric probabilistic k-nearest-neighbours classifier. In: Intelligent Data Engineering and Automated Learning-IDEAL, pp. 654-659 (2004)

7. Gionis, A., Indyk, P., Motwani, R.: Similarity search in high dimensions via hashing. In: Proceedings of the 25th Very Large Database (VLDB) Conference, pp. 518-529 (1999)

8. Grangier, D., Bengio, S.: A discriminative kernel-based model to rank images from text queries. IEEE Transactions on Pattern Analysis and Machine Intelligence (PAMI) 30(8), 1371-1384 (2008)

9. Guillaumin, M., Mensink, T., Verbeek, J., Schmid, C.: Tagprop: Discriminative metric learning in nearest neighbor models for image auto- annotation. In: International Conference on Computer Vision (2009)

10. Indyk, P.: Stable distributions, pseudorandom generators, embeddings, and data stream computation. J. ACM 53(3), 307-323 (2006)

11. Jeon, J., Lavrenko, V., Manmatha, R.: Automatic image annotation and retrieval using crossmedia relevance models. In: SIGIR 2003: Proceedings of the 26th annual international ACM SIGIR conference on Research and development in informaion retrieval, pp. 119-126. ACM, New York (2003)

12. Jeon, J., Manmatha, R.: Using maximum entropy for automatic image annotation. In: International Conference on Image and Video Retrieval, pp. 2040-2041 (2004)

13. Makadia, A., Pavlovic, V., Kumar, S.: A new baseline for image annotation. In: European Conference on Computer Vision (2008)

14. Manocha, S., Girolami, M.A.: An empirical analysis of the probabilistic k-nearest neighbour classifier. Pattern Recognition Letters 28(13), 1818-1824 (2007)

15. Monay, F., Gatica-Perez, D.: Plsa-based image auto-annotation: constraining the latent space. In: ACM Multimedia, pp. 348-351 (2004)

16. Naphade, M.: On supervision and statistical learning for semantic multimedia analysis. Journal of Visual Communication and Image Representation 15(3) (2004)

17. Pan, J.Y., Yang, H.J., Duygulu, P., Faloutsos, C.: Automatic image captioning. In: International Conference on Multimedia and Expo, ICME (2004)

18. Sivic, J., Zisserman, A.: Video google: A text retrieval approach to object matching in videos. In: ICCV (2003)

19. Vogel, J., Schiele, B.: Natural scene retrieval based on a semantic modeling step. In: Enser, P.G.B., Kompatsiaris, Y., O'Connor, N.E., Smeaton, A., Smeulders, A.W.M. (eds.) CIVR 2004. LNCS, vol. 3115, pp. 207-215. Springer, Heidelberg (2004) 